\title{
On finite-time stability of homogeneous systems with multiplicative bounded function*
}

\author{
Y. Braidiz ${ }^{1}$, A. Polyakov ${ }^{2}$, D. Efimov ${ }^{2}$ and W. Perruquetti ${ }^{1}$
}

\begin{abstract}
In this paper, we study the finite-time stability of a class of nonlinear systems $\dot{x}=f(x)=H(x) b(x)$, where $H$ is homogeneous and $b$ is bounded. We define the homogeneous extension of the non-homogeneous function $f$ and use this extension to prove that, under some conditions on $b$, if the system $\dot{x}=f(x)$ is globally asymptotically stable, then it is finite-time stable. An example of global asymptotic stable system with some additional conditions is presented in the last section to illustrate the obtained results.
\end{abstract}

\section{INTRODUCTION}

Stability is one of the main research directions in the mathematical study of dynamical systems and there is a large number of papers and books that have been published on the subject (see [1]-[4] and references therein). In general, there are different rates of convergence.

Over the years, researchers mainly focused their attention on asymptotic and exponential stability: trajectories converge to an equilibrium state in "infinite time" [4], [5]. However, in a lot of engineering domains, the time is a critical constraint which must be taken into account. Hence, in many applications, it is desirable to get the so called finite-time convergence of the solutions to an operating point. This possibility has been mentioned by Erugin 1951 and Zubov 1957, and studied by Roxin 1966, Korobov 1979 [6], [7], many useful properties or finite-time stable (FTS) systems can be found in [8]-[10]. There are two methods to establish FTS: Lyapunov function approach or homogeneity.

Investigating FTS of dynamical systems by using Lyapunov function approach was introduced in several works (see [6]-[14]). Proving FTS of dynamical systems and differential inclusion using smooth or non-smooth Lyapunov function was investigated in [12]. Some necessary and sufficient conditions to guarantee the FTS for autonomous scalar systems was presented in Haimo's paper [15]. This result was extended to $n$-dimensional systems in [11] through the use of Lyapunov functions as in [8]. Again, a necessary and sufficient conditions were developed for finite-time stabilization of systems like $\dot{x}=f(x)+g(x) u$ ( $u$ is the input) [11].

\footnotetext{
*This work was partially supported by ANR 15 CE23 0007 (Project Finite4SoS), the Government of Russian Federation (Grant 08-08) and the Ministry of Education and Science of Russian Federation (Project 14.Z50.31.0031).

${ }^{1}$ Y. Braidiz and W. Perruquetti are with Ecole Centrale, Univ. Lille, CNRS, UMR 9189 - CRIStAL, F-59000 Lille, France, email: $\quad$ youness.braidiz, wilfrid.perruquetti \}ecentralelille.fr.

${ }^{2}$ D. Efimov and A. Polyakov are with Inria, Univ. Lille, CNRS, UMR 9189 - CRIStAL, F-59000 Lille, France and with Department of Control Systems and Informatics, University ITMO, 197101 Saint Petersburg, Russia. email: \{denis.efimov, andrey.polyakov\}einria.fr.
}

We may also establish the FTS by using the so called homogeneity. This tool was used to prove different kinds of results. Particularly, in control theory, homogeneity has been introduced in order to investigate the stability and stabilization of dynamical systems [2], [1], [16].

FTS of homogeneous systems was studied by Zubov in the 1957 for standard homogeneity and extended by Bhat and Bernstein in [9] for weighted homogeneity (2005) and it also appeared with Rosier and Bacciotti in [3], where it has been proven that homogeneous systems of negative degree locally asymptotically stable (LAS) possess FTS property. This result was extended for differential inclusions (DIs) [17] together with Rosier's theorem [18]. The research about FTS of homogeneous evolution systems was also presented in [19] which uses a general definition of homogeneity in Banach and Hilbert spaces.

The novelty of this paper consists in a result about FTS of a class of a non-homogeneous systems of the form $\dot{x}=H(x) b(x)$, with $H$ is homogeneous and $b$ satisfies boundedness conditions. We extend the result that was proved for a GAS homogeneous system with negative degree of homogeneity. In particular, we prove that, with some sufficient conditions on the function $b$, we may guarantee the global FTS (GFTS) of the GAS system $\dot{x}=H(x) b(x)$. To do so, we use a homogeneous extension which is given by a homogeneous differential inclusion. The main peculiarity of a homogeneous extension is that it can be defined in the cases when homogeneous approximations do not exist. Then, using the results that were proven for GAS homogeneous differential inclusion with a negative degree of homogeneity (see for more details [20]), we will prove the FTS of the original dynamical system.

The paper is organized as follows. After introducing some notations and definitions about finite-time stability and homogeneity in Section II, we define the homogeneous extension of a non-homogenous continuous function $f$ : $\mathbb{R}^{n} \longrightarrow \mathbb{R}^{n}$, that we use, by giving a sufficient conditions on $f$, to prove the global FTS of a GAS system of the form $\dot{x}=$ $f(x)$ (in Section III and Section IV). In the last section we present one example which shows how to apply the obtained results. The aim of this paper is to investigate the GFTS of non-homogeneous differential equation using a constructed homogeneous differential inclusion and Lyapunov functions.

\section{PRELiminaries}

\section{A. Notations}

- $\mathbb{R}_{+}=\{x \in \mathbb{R}: x \geq 0\}$, where $\mathbb{R}$ is the set of real numbers. 
- $|\cdot|$ denotes the absolute value in $\mathbb{R},\|\cdot\|$ denotes the Euclidean norm in $\mathbb{R}^{n}$.

- $S=\left\{x \in \mathbb{R}^{n}:\|x\|=1\right\}$ denotes the unit sphere in $\mathbb{R}^{n} . B\left(x_{0}, r\right)=\left\{x \in \mathbb{R}^{n}:\left\|x-x_{0}\right\|<r\right\}$ denotes the open ball of radius $r>0$ centered at a point $x_{0}$.

- $\mathcal{M}_{m, n}$ is the set of all $m \times n$-matrices over the field of real numbers, and it forms a vector space. When $m=n$ we write $\mathcal{M}_{n}$ instead of $\mathcal{M}_{n, n}$.

- $\|\cdot\|_{\mathcal{M}_{m, n}}$ denotes the matrix norm induced by $\|\cdot\|$, i.e.

$$
\|A\|_{\mathcal{M}_{m, n}}=\sup _{x \in S}\|A x\|
$$

- A continuous function $\alpha: \mathbb{R}_{+} \longrightarrow \mathbb{R}_{+}$belongs to the class $\mathcal{K}$ if $\alpha(0)=0$ and the function is strictly increasing. The function $\alpha: \mathbb{R}_{+} \longrightarrow \mathbb{R}_{+}$belongs to the class $\mathcal{K}_{\infty}$ if $\alpha \in \mathcal{K}$ and it is increasing to infinity.

- The notation $\langle D V(x), f(x)\rangle$ stands for the directional derivative of a continuously differentiable function $V$ with respect to the vector field $f$ evaluated at point $x$.

- $\mathcal{C}^{p}\left(\mathbb{R}^{n}, \mathbb{R}\right)$ denotes the space of functions $f: \mathbb{R}^{n} \longrightarrow \mathbb{R}$ which have $p$ continuous derivatives and $\mathcal{C}^{\infty}\left(\mathbb{R}^{n}, \mathbb{R}\right)$ denotes the space of functions $f: \mathbb{R}^{n} \longrightarrow \mathbb{R}$ which are smooth.

- we denote by $\mathcal{C} \mathcal{L}^{0}(E, F)$ (respectively $\mathcal{C} \mathcal{L}^{k}(E, F)$ ) the set of continuous functions on $E$, locally Lipschitz on $E \backslash\{0\}$ with value in $F$ (respectively the set of continuous functions on $E, \mathcal{C}^{k}$ on $E \backslash\{0\}$ with value in $F$ ).

\section{B. Stability properties and generalized homogeneity}

In this subsection we give some definitions of homogeneity and FTS of a system:

$$
\left\{\begin{array}{l}
\dot{x}(t)=f(x(t)) \\
x(0)=x_{0} \in \mathbb{R}^{n}
\end{array}\right.
$$

where $x(t) \in \mathbb{R}^{n}$ is the state, $f: \mathbb{R}^{n} \longrightarrow \mathbb{R}^{n}, f(0)=0$, and denote the corresponding solution as $x\left(t, x_{0}\right)$.

1) Finite-time stability: Now let us give the definition of FTS which will be investigated in this paper.

Definition 2.1: [8], [21] Assume that the system (1) possesses unique solutions in forward time for all initial conditions except the origin. Then the origin of the system (1) is FTS, iff there exists a nonempty open neighborhood of the origin $\mathcal{V}$ in $\mathbb{R}^{n}$ such that:

- there exist a function $\alpha \in \mathcal{K}$ such that for all $x_{0} \in \mathcal{V}$ we have $\left\|x\left(t, x_{0}\right)\right\| \leq \alpha\left(\left\|x_{0}\right\|\right), \forall t \geq 0$.

- there exists a function $T: \mathcal{V} \longrightarrow \mathbb{R}^{+}$such that if $x_{0} \in \mathcal{V}$ then $\lim _{t \longrightarrow T\left(x_{0}\right)} x\left(t, x_{0}\right)=0 . T$ is called the settling-time of the system (1);

- If the settling-time function $T$ is bounded for all $x \in \mathcal{V}$, we say that our dynamical system is fixed-time stable (FxTS). We say that the ball $B$ is fixed-time stable for (1), if there exists $0<T<+\infty$ such that $x\left(t, x_{0}\right) \in$ $B, \forall t \geq T, \forall x_{0} \in \mathbb{R}^{n}$.

In addition, if $\mathcal{V}=\mathbb{R}^{n}$, then the origin is a globally FTS (GFTS).
The following proposition shows how to investigate the FTS by using Lyapunov theory.

Proposition 2.1: [13] Assume that the (1) has a unique solution in forward time outside the origin. The origin of this system is FTS with a continuous settling-time function at the origin if and only if there exists a real number $c>0, \alpha \in$ ]0,1[ and a class $\mathcal{C} \mathcal{L}^{\infty}$ Lyapunov function $V: \mathcal{V} \longrightarrow \mathbb{R}^{+}$ satisfying

$$
\langle D V(x), f(x)\rangle \leq-c[V(x)]^{\alpha}
$$

for all $x \in \mathcal{V}$.

2) Homogeneity: In control theory, homogeneity simplifies qualitative analysis of nonlinear dynamic systems. So that, it allows a local properties (e.g., local stability) to be extended globally using a property of the solutions (homogeneity is a special kind of Lie symmetry). In order to define this notion, let us introduce the notion of dilation group.

Definition 2.2: A map $\boldsymbol{d}: \mathbb{R} \longrightarrow \mathcal{M}_{n}(\mathbb{R})$ is called a dilation in $\mathbb{R}^{n}$ iff it satisfies

- Group property: $\boldsymbol{d}(0)=I_{n}, \boldsymbol{d}(t+s)=\boldsymbol{d}(t) \boldsymbol{d}(s), t, s \in$ $\mathbb{R}$.

- Continuity property: $\boldsymbol{d}$ is continuous, i.e. $\forall t>0, \forall \varepsilon>$ $0, \exists \gamma>0:|s-t|<\gamma \Longrightarrow\|\boldsymbol{d}(s)-\boldsymbol{d}(t)\|_{\mathcal{M}_{n}} \leq \varepsilon$

- Limit property: $\lim _{s \rightarrow-\infty}\|\boldsymbol{d}(s) x\|=0$ and $\lim _{s \rightarrow+\infty}\|\boldsymbol{d}(s) x\|=+\infty$ uniformly on the unite sphere.

Definition 2.3: - The dilation $\boldsymbol{d}$ is monotone if $\|\boldsymbol{d}(s)\|_{\mathcal{M}_{n}} \leq 1, \forall s \leq 0$

- The dilation $\boldsymbol{d}$ is strictly monotone in $\mathbb{R}^{n}$ if $\exists \beta>0$ such that $\|\boldsymbol{d}(s)\|_{\mathcal{M}_{n}} \leq e^{\beta s}, \forall s \leq 0$.

Property 2.1: [22], [23] The matrix $G_{\boldsymbol{d}} \in \mathcal{M}_{n}$ defined as $G_{\boldsymbol{d}}=\lim _{s \longrightarrow 0} \frac{\boldsymbol{d}(s)-I_{n}}{s}$ is known as the generator of the group $\boldsymbol{d}(s)$ and satisfies the following properties:

$$
\frac{d}{d s} \boldsymbol{d}(s)=G_{\boldsymbol{d}} \boldsymbol{d}(s)=\boldsymbol{d}(s) G_{\boldsymbol{d}} \text { and } \boldsymbol{d}(s)=e^{G_{\boldsymbol{d}} s}=\sum_{i=0}^{+\infty} \frac{s^{i} G_{\boldsymbol{d}}^{i}}{i !} .
$$

Let $S_{\mathbf{d}}(r)=\left\{x \in \mathbb{R}^{n}:\|\mathbf{d}(-\ln (r)) x\|=1\right\}, r>0$ is called the homogeneous sphere of radius $r$. If $r=1$ we write $S_{\mathbf{d}}$ instead of $S_{\mathbf{d}}(1)$. Now we will defined the canonical homogeneous norm.

Definition 2.4: A continuous function $p: \mathbb{R}^{n} \longrightarrow \mathbb{R}_{+}$is said to be d-homogeneous norm if $p(x) \longrightarrow 0$ as $x \longrightarrow 0$ and $p(\boldsymbol{d}(s) x)=e^{s} p(x)>0$ for $x \in \mathbb{R}^{n} \backslash\{0\}$ and $s \in \mathbb{R}$.

For monotone dilations the homogeneous norm $\|\cdot\|_{d}$ is called the canonical homogeneous norm

$$
\|x\|_{\boldsymbol{d}}=e^{s_{x}}, \text { where } s_{x} \in \mathbb{R}:\left\|\boldsymbol{d}\left(-s_{x}\right) x\right\|=1 .
$$

Definition 2.5: A vector field $f: \mathbb{R}^{n} \longrightarrow \mathbb{R}^{n}$ (resp., a function $h: \mathbb{R}^{n} \longrightarrow \mathbb{R}$ ) is said to be $d$ homogeneous of degree $\nu \in \mathbb{R}$ iff for all $s \in \mathbb{R}$ and all $x \in \mathbb{R}^{n}$ we have $e^{-\nu s} \boldsymbol{d}(-s) f(\boldsymbol{d}(s) x)=f(x)$, (resp., $\left.e^{-\nu s} h(\boldsymbol{d}(s))=h(x)\right)$.

Homogeneity property was introduced also for Banach spaces [19], [23], and it is given by a group of dilations 
in Banach or Hilbert space. The following lemma provides a useful comparaison between homogeneous functions.

Lemma 2.1: Suppose that $V_{1}$ and $V_{2}$ are continuous realvalued functions on $\mathbb{R}^{n}$, d-homogeneous of degrees $l_{1}>0$ and $l_{2}>0$, respectively, and $V_{1}$ is positive definite. Then, for every $x \in \mathbb{R}^{n}$ we have

$$
a_{1}\left[V_{1}(x)\right]^{\frac{l_{2}}{l_{1}}} \leq V_{2}(x) \leq a_{2}\left[V_{1}(x)\right]^{\frac{l_{2}}{l_{1}}},
$$

with $a_{1}=\min _{\left\{z: V_{1}(z)=1\right\}} V_{2}(z)$ and $a_{2}=\max _{\left\{z: V_{1}(z)=1\right\}} V_{2}(z)$.

The Lemma 2.1 has been proven in [9] for weighted homogeneous functions and we can use the same truck to prove it for $\mathbf{d}$-homogeneous functions $V_{1}$ and $V_{2}$. Hence, because $V_{1}>0$ then for every $x \in \mathbb{R}^{n} \backslash\{0\}, \exists z \in$ $\left\{y: V_{1}(y)=1\right\}$ such that $z=\mathbf{d}\left(-\ln \left(V_{1}(x)\right)\right) x$.

In the next section we will use the Lyapunov theory to show some robust stability results. The existence of a homogeneous Lyapunov function for a GAS homogeneous system was provided by Zubov in 1957, Rosier [18] and Polyakov [24], [22] by using weighted and generalized homogeneity.

\section{HomogeneOUS EXTENSION.}

In this section, we define a homogeneous extension which allows some homogeneity methods to be applied for nonhomogeneous systems (see [23]). This extension is given by a differential inclusion.

Differential inclusions appear as a model of dynamical systems which do not satisfy the classical assumptions of regularity. Regularization of the system (1) proposed by Filippov [25] consists in replacing (1) with a suitable differential inclusion

$$
\dot{x} \in F(x), x \in \mathbb{R}^{n}, F: \mathbb{R}^{n} \longrightarrow 2^{\mathbb{R}^{n}} .
$$

The notions of solution obtained in this way depend on the construction of the set valued map $F$. If the set valued map $F(x)$ is upper semicontinuous, compact and convex valued, and locally bounded, then (5) has solutions.

Definition 3.1: Homogeneous extension of a function $f$ : $\mathbb{R}^{n} \longrightarrow \mathbb{R}^{n}$ is a set valued d-homogeneous map $F: \mathbb{R}^{n} \rightrightarrows$ $\mathbb{R}^{n}$, such that $f(x) \in F(x)$ for any $x \in \mathbb{R}^{n}$ and $F(x)$ is bounded for every fixed $x \in \mathbb{R}^{n}$.

This extension appears, for example, as a result of Filippov regularization of a homogeneous discontinuous vector field (see [26]). In this paper the set-valued map $F$ is defined by

$$
F(x)=\bigcup_{s \in \mathbb{R}}\left\{e^{-\nu s} \mathbf{d}(-s) f(\mathbf{d}(s) x)\right\}, x \in \mathbb{R}^{n},
$$

where $\mathbf{d}$ is a generalized dilation and $\nu \in \mathbb{R}$. $F$ is the homogeneous extension (see [23]) of the continuous function $f$. Indeed, this set valued map $F$ is d-homogeneous in the following sense

$$
e^{\nu s} \mathbf{d}(s) F(x)=F(\mathbf{d}(s) x), s \in \mathbb{R}, x \in \mathbb{R}^{n} .
$$

Another goal for which we define this extension is that there exist some functions which do not have a homogeneous approximation. Therefore, to study its stability and to evaluate its rate of convergence the homogeneous extension can be used. The investigation of this issue is presented in the rest of this section.

An examlpe of such a system is the following:

$$
\dot{x}(t)=-\left(2+\cos \left(\frac{1}{x(t)}\right)\right) x(t), t \geq 0 .
$$

The right-hand side of (8) does not have a homogeneous approximation, but it has a homogenous extension, which will allow us to study the behaviour of its solution: $\dot{x}(t) \in$ $F(x(t))=-x(t)[1,3], t \geq 0$.

Remark 3.1: We mention that we do not need to prove the existence of a solution to the extended inclusion $\dot{x} \in F(x)$, where $F$ is given by (6), since $f(x) \in F(x)$ and solution of $\dot{x}=f(x)$ always exists if $f$ is continuous: the solution of the system (1) belongs to the set of the solutions of the differential inclusion $\dot{x} \in F(x)$. The idea of this paper is to study the FTS of the differential inclusion (5), with $F$ defined by (6), by using the Lyapunov theory.

If (5), where $F$ defined by (6), is GAS, then (1) is also GAS. It is interesting to know when the inverse result holds. Thus, we choose the function $f$ such that the system (1) has a solution. The function $f$ of the system (1) satisfies the following assumptions

$\mathcal{H}_{1}: f: x \in \mathbb{R}^{n} \longmapsto f(x)=H(x) b(x) \in \mathbb{R}^{n}$.

$\mathcal{H}_{2}: H: \mathbb{R}^{n} \longrightarrow \mathcal{M}_{n, m}$ is continuous and d-homogeneous of degree $\nu$ (for every $b \in \mathbb{R}^{m}$ the function $x \longmapsto$ $H(x) b$ is d-homogeneous of degree $\nu$, see below) and $H(x)=0 \Leftrightarrow x=0$.

$\mathcal{H}_{3}: b: \mathbb{R}^{n} \longrightarrow \mathbb{R}^{m}$ is bounded $(\exists M>0,\|b(x)\| \leq$ $\left.M, \forall x \in \mathbb{R}^{n}\right)$ and $b: \mathbb{R}^{n} \backslash\{0\} \longrightarrow \mathbb{R}^{m}$ is continuous.

$\mathcal{H}_{4}$ : The system (1) is GAS.

In particular, the hypothesis $\mathcal{H}_{4}$ implies that $b(x)$ does not belong to the kernel of $H(x), \forall x \in \mathbb{R}^{n} \backslash\{0\}$. By the definition of the function $f(x)=H(x) b(x) \in \mathbb{R}^{n}$, since $b$ is bounded, then the set $F(x)$ is also bounded for any fixed $x$. This ensures that the supremum of $F$ on $S$ is finite. In the following theorem we give a sufficient condition which guarantee GAS of the differential inclusion $\dot{x} \in F(x)$.

Theorem 3.1: Let

- the system (1) satisfies the assumptions $\mathcal{H}_{1}, \mathcal{H}_{2}, \mathcal{H}_{3}$ and $\mathcal{H}_{4}$,

- the dilation $\boldsymbol{d}$ is strictly monotone,

- there exist a Lyapunov function $V$ of the system (1) and $0<\beta<1$, such that

$$
\max _{\|x\|_{d}=1}\|b(y)-b(x)\| \leq \frac{-\beta \max _{\|x\|_{d}=1}\langle D V(x), f(x)\rangle}{\max _{\|x\|_{d}=1}\|H(x)\|_{\mathcal{M}_{n, m}} \max _{\|x\|_{d}=1}\|D V(x)\|},
$$

$\forall y \in \mathbb{R}^{n} \backslash\{0\}$

Then the differential inclusion (5), with $F$ is defined by (6), is GAS and there exists a d-homogeneous positive definite Lyapunov function $W$ such that

$\langle D W(x), h\rangle<0, \forall h \in F(x)$, and $x \in \mathbb{R}^{n} \backslash\{0\} . \quad$ (10) All proofs are excluded due to space limitations. The following Remark treats the case when a nonlinear dynamical systems cannot satisfy the condition (9). 
Remark 3.2: If the function $b$ satisfies the condition

- $\max _{\|x\|_{d}=1}\|b(x)\| \leq \max _{\|x\|_{d}=1}\|b(y)-b(x)\|, \forall y \in \mathbb{R}^{n} \backslash\{0\}$.

Then the system (1) with the assumptions $\mathcal{H}_{1}, \mathcal{H}_{2}, \mathcal{H}_{3}$ and $\mathcal{H}_{4}$, will not satisfy the condition (9).

Next, we consider the simple case when the function $b$ is a scalar function.

Corollary 3.1: If the vector fieled $f$ of the system (1) is defined by $f(x)=H(x) b(x)$, such that the function $b$ : $\mathbb{R}^{n} \longrightarrow \mathbb{R}$, is continuous, bounded and $H: \mathbb{R}^{n} \longrightarrow \mathbb{R}^{n}$ is continuous, $\boldsymbol{d}$-homogeneous with degree of homogeneity $\nu$. We assume that the system (1) is GAS, and $b(x)=0 \Longrightarrow$ $x=0$. Then the differential inclusion (5), with $F$ is defined by (6), is GAS.

\section{FINITE TIME STABILITY RESUlTS}

The following theorem shows the FTS of the system (1) when the degree of homogeneity of $H$ is negative and (9) is satisfied.

Theorem 4.1: If

- the system (1) satisfies the assumptions $\mathcal{H}_{1}, \mathcal{H}_{2}, \mathcal{H}_{3}$ and $\mathcal{H}_{4}$

- the dilation $\boldsymbol{d}$ is strictly monotone,

- the degree of homogeneity of $H$ is negative $(\nu<0)$ and (9) holds.

Then the system (1) is GFTS. If the two first assumptions hold and the degree of homogeneity of $H$ is positive $(\nu>0)$. Then the system (1) is fixed-time convergent to a ball.

Remark 4.1: - If the vector fieled $f$ of the system (1) ensure the boundedness of the homogeneous extension $F(x)$ for every fixed $x \in \mathbb{R}^{n}$, the dilation $\boldsymbol{d}$ is strictly monotone, the degree of homogeneity of $F$ is negative and there exist a Lyapunov function $V$ of the system (1) and $0<\beta<1$, such that

$$
\begin{gathered}
\max _{\|x\|_{d}=1}\left\|e^{-\nu \tau} \boldsymbol{d}(-\tau) f(\boldsymbol{d}(\tau) x)-f(x)\right\| \\
\leq \frac{-\beta \max _{\|x\|_{d}=1}\langle D V(x), f(x)\rangle}{\max _{\|x\|_{\boldsymbol{d}}=1}\|D V(x)\|}
\end{gathered}
$$

$\forall \tau \in \mathbb{R}$. Then the system (1) is GFTS.

- If the degree of homogeneity of $F$ is positive, then we may guarantee the fixed-time convergence of the system (1) to a ball.

- If $\max _{\|x\|_{d}=1}\|f(x)\| \leq \max _{\|x\|_{d}=1}\left\|e^{-\nu \tau} \boldsymbol{d}(-\tau) f(\boldsymbol{d}(\tau) x)-f(x)\right\|$, $\forall \tau \in \mathbb{R}$. Then the condition (11) will not hold.

Corollary 4.1: If the vector fieled $f$ of the system (1) is defined by $f(x)=H(x) b(x)$, such that the function $b$ : $\mathbb{R}^{n} \longrightarrow \mathbb{R}$, is continuous, bounded and $H: \mathbb{R}^{n} \longrightarrow \mathbb{R}^{n}$ is continuous, $\boldsymbol{d}$-homogeneous with degree of homogeneity $\nu<0$. We assume that the system (1) is GAS, and $b(x)=$ $0 \Longrightarrow x=0$. Then the sytem (1) is GFTS.

Example 4.1: Let us consider the following example:

$$
f: x \in \mathbb{R} \longmapsto-\left(1+0.5 \cos \left(\frac{1}{x}\right)\right) x^{\frac{2}{3}} \in \mathbb{R} .
$$

Let $F(x)=\bigcup_{s \in \mathbb{R}}\left\{e^{-s} \boldsymbol{d}(-s) f(\boldsymbol{d}(s) x)\right\}$ be the homogeneous extension of the function $f$, with $\boldsymbol{d}(s)=e^{3 s}$. Then $F(x)=$ $\left.-x^{\frac{2}{3}}\right] \frac{1}{2}, \frac{3}{2}[$. For a fixed $x, F(x)$ is bounded. The system $\dot{x}(t)=f(x(t))$ is GAS, then there exists a Lyapunov function $V$ fior the system (1). From the definition of $f$, one has

$$
e^{\tau} \boldsymbol{d}(-\tau) f(\boldsymbol{d}(\tau) x)=-\left(1+0.5 \cos \left(\frac{1}{\boldsymbol{d}(\tau) x}\right)\right) x^{\frac{2}{3}}
$$

The fact that $\frac{\left(1+0.5 \cos \left(\frac{1}{d(\tau) x}\right)\right)}{\left(1+0.5 \cos \left(\frac{1}{x}\right)\right)}>0, \forall x \in \mathbb{R}^{*}$ and $\forall \tau \in \mathbb{R}$, gives

$$
\begin{aligned}
& \frac{\partial V(x)}{\partial x} e^{\tau} \boldsymbol{d}(-\tau) f(\boldsymbol{d}(\tau) x) \\
& =\frac{\partial V(x)}{\partial x} f(x) \frac{e^{\tau} \boldsymbol{d}(-\tau) f(\boldsymbol{d}(\tau) x)}{f(x)} \\
& =\frac{\partial V(x)}{\partial x} f(x) \frac{\left[-\left(1+0.5 \cos \left(\frac{1}{\boldsymbol{d}(\tau) x}\right)\right) x^{\frac{2}{3}}\right]}{\left[-\left(1+0.5 \cos \left(\frac{1}{x}\right)\right) x^{\frac{2}{3}}\right]} \\
& =\frac{\partial V(x)}{\partial x} f(x) \frac{\left(1+0.5 \cos \left(\frac{1}{\boldsymbol{d}(\tau) x}\right)\right)}{\left(1+0.5 \cos \left(\frac{1}{x}\right)\right)}<0 .
\end{aligned}
$$

Which proves the GAS of the origin under the set valued $F(x)$. This inequality and the fact that the degree of homogeneity $\nu=-1$ imply that the system $\dot{x}=f(x)$ is globally FTS.

\section{ACADEMIC EXAMPLE}

Let us illustrate this results by the following example, for $\alpha, \beta>0$

$$
\left\{\begin{array}{l}
\dot{x}=y-x^{\frac{2}{3}} \operatorname{sign}(x) \\
\dot{y}=-\alpha x^{\frac{1}{3}}-\beta\left(2-\cos \left(\frac{1}{\sqrt{x^{2}+y^{2}}}\right)\right)|y|^{\frac{1}{2}} \operatorname{sign}(y)
\end{array}\right.
$$

Again, we can write the system (15), as the following

$$
\dot{z}=H(z) b(z), z=\left(\begin{array}{l}
x \\
y
\end{array}\right)
$$

with

$$
H(x, y)=\left(\begin{array}{cc}
y-x^{\frac{2}{3}} \operatorname{sign}(x) & 0 \\
-\alpha x^{\frac{1}{3}} & -\beta|y|^{\frac{1}{2}} \operatorname{sign}(y)
\end{array}\right)
$$

and

$$
b(x, y)=\left(\begin{array}{c}
1 \\
2-\cos \left(\frac{1}{\sqrt{x^{2}+y^{2}}}\right)
\end{array}\right) .
$$

The function $H$ is continuous and $\mathbf{d}$-homogeneous of degree of homogeneity $\nu=-1$, provided that the dilation $\mathbf{d}$ is defined as follows $\mathbf{d}(s)=\left(\begin{array}{ll}e^{3 s} & 0 \\ 0 & e^{2 s}\end{array}\right)$, which corespond to a weighted homogeneity. The function $b$ is continuous and bounded. We use the Lyapunov function

$$
V(x, y)=\alpha \frac{3}{4} x^{\frac{4}{3}}+\frac{1}{2} y^{2}
$$




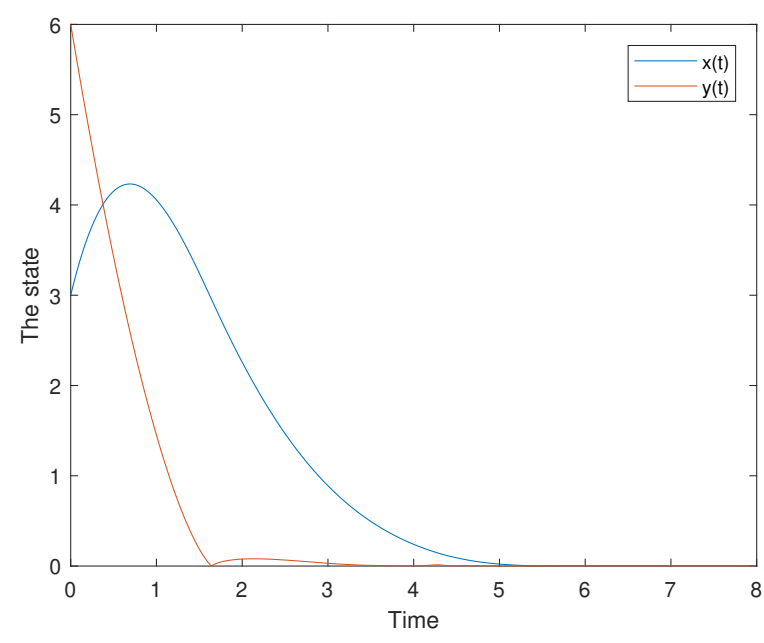

Fig. 1. The solutions of the system (15) with $\alpha=0.5, \beta=2$ and the initial condition $\left(x_{0}, y_{0}\right)=(3,6)$.

one gets $\dot{V}=-\alpha|x|-\beta\left(2-\cos \left(\frac{1}{\sqrt{x^{2}+y^{2}}}\right)\right)|y|^{\frac{3}{2}}$. Then, we deduce that the origin of system (15) is GAS. The homogeneous extension of the function $f$ is given by

$$
F(z)=\bigcup_{s \in \mathbb{R}}\{H(z) b(\mathbf{d}(s) z)\} .
$$

Again by using the homogeneous Lyapunov function $V$, one has

$$
\begin{aligned}
& \langle D V(z), H(z) b(\mathbf{d}(s) z)\rangle \\
& =-\alpha|x|-\beta\left(2-\cos \left(\frac{1}{\sqrt{e^{6 s} x^{2}+e^{4 s} y^{2}}}\right)\right)|y|^{\frac{3}{2}}<0,
\end{aligned}
$$

$\forall z \in S_{\mathbf{d}}$ and $\forall s \in \mathbb{R}$. This proves that the differential inclusion given by the homogenous extension (18) is GAS, and the degree of homogeneity $\nu=-1<0$, then the theorem (3.1) implies that the origin of system (15) is FTS. The Figure 1 shows the solution of the system (16) with initial condition $(3,2)$ which reach the origin in finite time (less than $t=6$ ).

\section{CONCLUSION}

Finite-time stability of a non-homogeneous GAS system (homogenoeus system with multiplicative bounded function) is investigated using the homogeneous extension and Lyapunov approach. Specifically, we presented some sufficient conditions on the vector fields to guarantee the finite-time stability of dynamical systems which are not homogeneous. By using the results that were proved for a homogeneous systems [9] we showed the FTS of our system. Example from Section V demonstrates that we can use the homogeneous extension to study the FTS of dynamical systems, which do not admit a homgeneous approximation. The homogeneous extension is also useful for stabilizing in finite-time a nonhomogenous evolution equations. This can be considered as a possible direction for the future research.

\section{REFERENCES}

[1] Wolfgang Hahn, Hans H Hosenthien, and H Lehnigk. Theory and application of Lyapunov's direct method. Prentice-Hall Englewood Cliffs, 1963.

[2] Wolfgang Hahn. Stability of motion, volume 138. Springer, 1967.

[3] Andrea Bacciotti and Lionel Rosier. Liapunov functions and stability in control theory. Springer Science \& Business Media, 2006.

[4] Taro Yoshizawa. Stability theory by Lyapunov's second method. 1966.

[5] Earl A Coddington and Norman Levinson. Theory of ordinary differential equations. Tata McGraw-Hill Education, 1955.

[6] E. Roxin. On finite stability in control systems. Rendiconti del Circolo Matematico di Palermo, 15(3):273-283, 1966.

[7] V.I. Korobov. A general approach to synthesis problem. Doklady Academii Nauk SSSR, 248:1051-1063, 1979.

[8] Sanjay P Bhat and Dennis S Bernstein. Finite-time stability of continuous autonomous systems. SIAM Journal on Control and Optimization, 38(3):751-766, 2000.

[9] Sanjay P. Bhat and Dennis S. Bernstein. Geometric homogeneity with applications to finite-time stability. Mathematics of Control, Signals and Systems, 17(2):101-127, 2005.

[10] Wilfrid Perruquetti, Thierry Floquet, and Emmanuel Moulay. Finitetime observers: application to secure communication. IEEE Transactions on Automatic Control, 53(1):356-360, 2008.

[11] Emmanuel Moulay and Wilfrid Perruquetti. Finite time stability of nonlinear systems. In Decision and Control, 2003. Proceedings. 42nd IEEE Conference on, volume 4, pages 3641-3646. IEEE, 2003.

[12] Emmanuel Moulay and Wilfrid Perruquetti. Finite time stability of differential inclusions. IMA Journal of Mathematical Control and Information, 22(4):465-475, 2005.

[13] Emmanuel Moulay and Wilfrid Perruquetti. Finite time stability and stabilization of a class of continuous systems. Journal of Mathematical Analysis and Applications, 323(2):1430-1443, 2006.

[14] Emmanuel Bernuau, Denis Efimov, Wilfrid Perruquetti, and Andrey Polyakov. On homogeneity and its application in sliding mode control. Journal of the Franklin Institute, 351(4):1866-1901, 2014.

[15] Varda Tepper Haimo. Finite time controllers. SIAM Journal on Control and Optimization, 24(4):760-770, 1986.

[16] Jean-Michel Coron. Control and nonlinearity. Number 136. American Mathematical Soc., 2007.

[17] Emmanuel Bernuau, Denis Efimov, Wilfrid Perruquetti, and Andrey Polyakov. On an extension of homogeneity notion for differential inclusions. In Control Conference ECC, 2013 European, pages 2204 2209. IEEE, 2013.

[18] Lionel Rosier. Homogeneous Lyapunov function for homogeneous continuous vector field. Systems \& Control Letters, 19(6):467-473, 1992.

[19] Andrey Polyakov, Denis Efimov, Emilia Fridman, and Wilfrid Perruquetti. On homogeneous distributed parameter systems. IEEE Transactions on Automatic Control, 61(11):3657-3662, 2016.

[20] Arie Levant, Denis Efimov, Andrey Polyakov, and Wilfrid Perruquetti. Stability and robustness of homogeneous differential inclusions. In Decision and Control (CDC), 2016 IEEE 55th Conference on, pages 7288-7293. IEEE, 2016.

[21] Yiguang Hong, Zhong-Ping Jiang, and Gang Feng. Finite-time input-to-state stability and applications to finite-time control. IFAC Proceedings Volumes, 41(2):2466-2471, 2008.

[22] Andrey Polyakov. Quadratic-like stability of nonlinear homogeneous systems. In 56th IEEE Conference on Decision and Control, 2017.

[23] Andrey Polyakov, Jean-Michel Coron, and Lionel Rosier. On homogeneous finite-time control for linear evolution equation in Hilbert space. IEEE Transactions on Automatic Control, 2018.

[24] Andrey Polyakov. Sliding mode control design using canonical homogeneous norm. International Journal of Robust and Nonlinear Control, 2018.

[25] Aleksej Fedorovič Filippov. Differential equations with discontinuous righthand sides: control systems, volume 18. Springer Science \& Business Media, 2013.

[26] Arie Levant. Homogeneity approach to high-order sliding mode design. Automatica, 41(5):823-830, 2005. 